\title{
Effect of fibroblast growth factor NV1FGF on amputation and death: a randomised placebo-controlled trial of gene therapy in critical limb ischaemia
}

\author{
Jill Belch, William R Hiatt, Iris Baumgartner, I Vickie Driver, Sigrid Nikol, Lars Norgren, Eric Van Belle, on behalf of the TAMARIS Committees \\ and Investigators
}

\section{Summary}

Background Patients with critical limb ischaemia have a high rate of amputation and mortality. We tested the hypothesis that non-viral 1 fibroblast growth factor (NV1FGF) would improve amputation-free survival.

Methods In this phase 3 trial (EFC6145/TAMARIS), 525 patients with critical limb ischaemia unsuitable for revascularisation were enrolled from 171 sites in 30 countries. All had ischaemic ulcer in legs or minor skin gangrene and met haemodynamic criteria (ankle pressure $<70 \mathrm{~mm} \mathrm{Hg}$ or a toe pressure $<50 \mathrm{~mm} \mathrm{Hg}$, or both, or a transcutaneous oxygen pressure $<30 \mathrm{~mm} \mathrm{Hg}$ on the treated leg). Patients were randomly assigned to either NV1FGF at $0.2 \mathrm{mg} / \mathrm{mL}$ or matching placebo (visually identical) in a 1:1 ratio. Randomisation was done with a central interactive voice response system by block size 4 and was stratified by diabetes status and country. Investigators, patients, and study teams were masked to treatment. Patients received eight intramuscular injections of their assigned treatment in the index leg on days $1,15,29$, and 43 . The primary endpoint was time to major amputation or death at 1 year analysed by intention to treat with a log-rank test using a multivariate Cox proportional hazard model. This trial is registered with ClinicalTrials.gov, number NCT00566657.

Findings 259 patients were assigned to NV1FGF and 266 to placebo. All 525 patients were analysed. The mean age was 70 years (range 50-92), 365 (70\%) were men, 280 (53\%) had diabetes, and $248(47 \%)$ had a history of coronary artery disease. The primary endpoint or components of the primary did not differ between treatment groups, with major amputation or death in 86 patients $(33 \%)$ in the placebo group, and $96(36 \%)$ in the active group (hazard ratio $1 \cdot 11,95 \%$ CI $0 \cdot 83-1 \cdot 49 ; \mathrm{p}=0 \cdot 48)$. No significant safety issues were recorded.

Interpretation TAMARIS provided no evidence that NV1FGF is effective in reduction of amputation or death in patients with critical limb ischaemia. Thus, this group of patients remains a major therapeutic challenge for the clinician.

Funding Sanofi-Aventis, Paris, France.

\section{Introduction}

Peripheral artery disease $\mathrm{e}^{1,2}$ affects up to 20 million individuals in North America and Europe, with 2-5\% developing the most severe form of the disease, critical limb ischaemia. This disorder is most often caused by atherosclerosis, but has a worse natural history than that of patients presenting with stable coronary or cerebrovascular disease. Although outcome is poorly documented, studies have supported the contention that prognosis is poor. ${ }^{3-6}$ For example, $46 \%$ of patients unsuitable for revascularisation will have major amputation within 12 months, ${ }^{3}$ with the substantial morbidity that this procedure entails. 2 years after a belowknee amputation, no more than $40 \%$ of the amputees will have reached full mobility, $15 \%$ will have had contralateral amputation and $15 \%$ an above-knee amputation, and $30 \%$ will have died. ${ }^{4}$ Results from studies ${ }^{5,6}$ have shown that the quality of life of a patient with critical limb disease equates to cancer patients with terminal disease.

In 1991, the European Working Group on Critical Limb Ischaemia concluded that no medical treatment had been shown to alter this natural history, ${ }^{7}$ and little progress has been reported since then. Despite the success of limb salvage with leg bypass, this procedure remains associated with a substantial morbidity and mortality, ${ }^{8}$ with the need for subsequent surgery and hospitalisation for wound complications as high as 50\%. Furthermore, in a substantial proportion of patients, poor general health, or lack of a suitable vein for revascularisation precludes open surgery, and the multifocal distribution and extent of the arterial occlusive disease might also make both open surgical or endovascular revascularisation impossible. At present, no effective alternatives to either percutaneous or surgical revascularisation in patients with critical limb ischaemia exist. ${ }^{9,10}$ Techniques to improve the perfusion of the ischaemic leg by less invasive means are needed. Substantial research has focused on development of therapeutic angiogenesis. In a meta-analysis ${ }^{11}$ of trials that included gene and cell-based therapies in peripheral artery disease, the investigators concluded that these therapies had the potential for clinical benefit.

Fibroblast growth factor type 1 (FGF1) modulates and enhances new blood-vessel formation ${ }^{12}$ and activates
Lancet 2011; 377: 1929-37

Published Online May 31, 2011 DOI:10.1016/501406736(11)60394-2

See Comment page 1894

The Institute of Cardiovascular Research, Vascular and Inflammatory Diseases Research Unit, Ninewells Hospital and Medical School, Dundee, UK (ProfJ Belch MD); Division of Cardiology, University of Colorado School of Medicine, Aurora, CO, USA (W R Hiatt MD); CPC Clinical Research, Aurora, CO, USA (W R Hiatt); Swiss Cardiovascular Centre, Division of Angiology, Inselspital, Bern University Hospital, Bern, Switzerland (I Baumgartner MD); Clinical Research Foot Care, Endovascular and Vascular Services, Boston University School of Medicine and Boston University Medical Centre, Boston, MA, USA (IV Driver DPM); Department of Angiology, Askleplios Klinik St Georg, Hamburg, Germany (S Nikol MD); Department of Surgery, Örebro University Hospital, Örebro, Sweden (L Norgren MD); and Department of Cardiology, Centre Hospitalier Régional Universitaire de Lille, and EA 2693, Université Lille Nord de France, Lille, France (Prof E Van Belle MD)

Correspondence to: Prof Jill J F Belch, Institute of Cardiovascular Research Ninewells Hospital and Medical School, Dundee DD1 9SY, UK j.j.f.belch@dundee.ac.uk 
migration, proliferation, and differentiation of endothelial cells, which result in sprouting of capillaries from pre-existing vessels. Non-viral 1 (NV1) FGF (riferminogene pecaplasmid), a naked DNA plasmid that includes the gene encoding for human FGF1, given intramuscularly into the calf and thigh leads to expression of human FGF1 protein. Results from studies in human beings of intramuscular administrations of NV1FGF showed expression of FGF1, and those from studies in animals ${ }^{12-14}$ showed restoration of a functional vascular network near the site of administration.

Panel 1: Major inclusion and exclusion criteria

\section{Inclusion criteria}

- Age $>50$ years

- Critical limb ischaemia with skin lesions (ischaemic ulcer[s] or minor gangrene)

- Objective evidence of critical limb ischaemia, including ankle systolic pressure $<70 \mathrm{~mm} \mathrm{Hg}$, or toe systolic pressure $<50 \mathrm{~mm} \mathrm{Hg}$ or $\mathrm{TcPO}_{2}<30 \mathrm{~mm} \mathrm{Hg}$

- Patent femoral artery inflow assessed by digital angiography, magnetic resonance, or $\mathrm{CT}$ angiography (doppler ultrasonography if a previous angiography is available) $<6$ months before first administration of study treatment

- Unsuitable for standard revascularisation as assessed by a vascular surgeon

- Negative screening for cancer (including family history, a complete physical examination of every system organ including the skin, a haematological blood test, a chest radiography, a stool haemoccult test, and, in the 6 months before screening, a measurement of prostate-specific antigen for men and a mammography and a Papanicolaou test for women, and any investigation required by national guidelines for cancer screening)

Exclusion criteria

- Previous major amputation on the leg to be treated

- Planned major amputation within the first month after randomisation

- Infected gangrene affecting the forefoot evidenced by imaging (radiography)

- Critical limb ischaemia caused by Buerger's disease

- Ulcers from venous or neuropathic origin if not associated with at least one ulcer from arterial origin

- Successful revascularisation procedure of the lower leg, or any other successful treatment (eg, neurostimulation) of the leg to be treated $<3$ months before randomisation

- Uncontrolled blood pressure defined as systolic blood pressure $\geq 180 \mathrm{~mm} \mathrm{Hg}$ or diastolic blood pressure $\geq 110 \mathrm{~mm} \mathrm{Hg}$

- Patient with a severe comorbid disorder, not expected to survive more than 12 months

- Acute cardiovascular events (eg, myocardial infarction, stroke, recent coronary intervention) within 3 months before randomisation

- Active or proliferative retinopathy and severe macular oedema

- Previous or present history of malignant disease, other than basal-cell carcinoma and cervical carcinoma in situ, within the past 5 years; previous malignant disease with relapse or therapy within the past 5 years

- Previous treatment with systemic growth angiogenic factors or with stem-cell therapy

- Women pregnant or breastfeeding, or of childbearing potential not protected by an effective contraceptive method of birth control; men not following effective contraceptive method with their partner of childbearing potential during the course of the study

$\mathrm{TcPO}_{2}=$ transcutaneous oxygen pressure
In an open label phase 1 trial of 51 patients, ${ }^{15}$ one intramuscular administration of NV1FGF in patients with critical limb ischaemia significantly improved symptoms (pain, ulcer size) and haemodynamic variables in the treated limb. ${ }^{15}$ In the phase 2 TALISMAN trial, ${ }^{16}$ administration of NV1FGF ( $4 \mathrm{mg}$ every 2 weeks) showed a similar improvement in ulcer healing (the primary endpoint) to that of placebo, but a $62.9 \%$ reduction of the risk of major amputation $(\mathrm{p}=0 \cdot 015)$ and a $56 \%$ reduction of the risk of major amputation or death $(\mathrm{p}=0.009)$ at 12 months as compared with placebo.

The main objective of this study was to show the clinical benefit of NV1FGF in delay of the time to major amputation or death in patients with clinical limb ischaemia with non-healing ischaemic skin lesions, in whom revascularisation was not possible.

\section{Methods \\ Patients}

This TAMARIS study is a multinational, double-blind, placebo-controlled phase 3 randomised gene therapy trial (EFC6145), in critical limb ischaemia. Trial recruitment was from Dec 1, 2007, to July 31, 2009 in 171 hospitals in 30 countries. For enrolment, patients had to have critical limb ischaemia with ischaemic lesions (Fontaine stage IV), and the diagnosis confirmed by at least one haemodynamic measurement (ankle pressure $<70 \mathrm{~mm} \mathrm{Hg}$ or toe pressure $<50 \mathrm{~mm} \mathrm{Hg}$, or transcutaneous oxygen pressure $\left[\mathrm{TcPO}_{2}\right]$ $<30 \mathrm{~mm} \mathrm{Hg}$ ) and by one imaging technique (angiography or doppler examination). Vascular surgeons had to confirm that patients were unsuitable for revascularisation and to justify this decision to the independent adjudication panel (event adjudication committee). Panel 1 shows the major inclusion and exclusion criteria. The research protocol was approved by the relevant institutional review boards or ethics committees and all patients gave written informed consent.

\section{Randomisation and masking}

Eligible patients were randomly assigned to receive either NV1FGF or placebo (ratio 1:1). Randomisation was stratified by diabetes status and by country and managed centrally by a central interactive voice response system (IVRS). The randomisation code list, with a block size of four, was generated by the sponsor with an electronic technique. The investigator, patient, and sponsor study teams were masked to treatment. Both the active compound (NV1FGF) and the placebo (saline solution) were similar in their appearance (transparent solution) and could not be distinguished from one another.

\section{Procedures}

If the disease affected both legs and both were unsuitable for revascularisation, the leg with the lowest pressure index (ankle brachial index or toe brachial index) was identified as the leg to be treated (index leg). The data for the other leg were recorded in the case-report form but not 


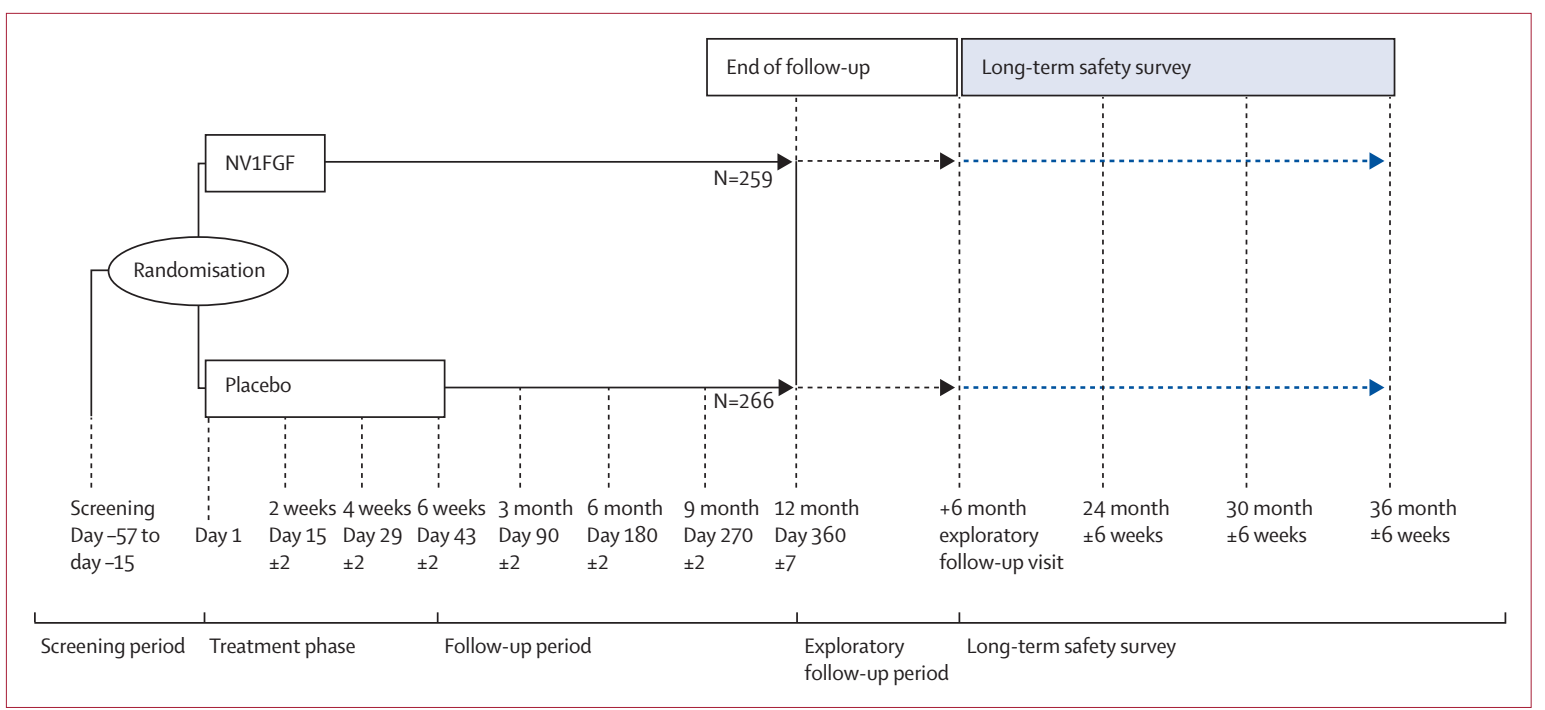

Figure 1: Study design of the EFC6145/TAMARIS trial

NV1FGF=non-viral 1 fibroblast growth factor.

analysed for efficacy. To aid standardisation, the methods used to assess skin lesions were clearly defined in the case-record form, which contained observer descriptions of ulcer sites, sizes, depths, texture (dry, moist, or gangrenous), and description of lesion management. Four treatment sessions were done 2 weeks apart. At every session, eight intramuscular injections of $0.5 \mathrm{mg}$ NV1FGF each were given in the leg to be treated: four into the calf muscle covering the anterior and posterior region and four into the thigh muscle of the ischaemic leg. The injection sites were selected according to an accessible good striated muscle mass and as close as possible to areas of known collateral blood flow development. In patients with poor muscle mass, the investigator was allowed to identify the injection sites using B-mode ultrasound. Investigators were advised to use present guidelines for optimum medical management of patients.

Figure 1 shows the study design. All patients were reviewed at 2, 4, and 6 weeks during the treatment phase, and then at 3, 6, 9, and 12 months in the observation phase for assessment of both efficacy and safety. An exploratory extended observation phase to assess long-term safety is in progress, in which the patients will be reviewed at $18,24,30$, and 36 months.

\section{Endpoints}

The primary combined endpoint of the trial was time to major amputation (above the ankle) of the treated leg or death from any cause in the study period of 12 months. All amputations were documented by anatomical level and indication (continuing ischaemia detrimental to the patient's health; presence of a lesion considered unlikely to heal; substantial infection, gangrene, or osteomyelitis such that the patient's life was endangered; and intolerable pain).
The main secondary endpoints were major amputation and death from any cause in the study period of 12 months analysed separately. Other secondary endpoints were: (1) all amputations, including all minor and major amputations on the treated leg; (2) skin lesion status (worsened, unchanged, improved, fully healed lesion); (3) pain intensity as assessed at rest (VAS Scale), ${ }^{17}$ (4) functionality and general health assessmentambulatory function and residential status for patients according to the Deneuville questionnaire, ${ }^{15}$ and overall quality of life as assessed with the EuroQoL questionnaire; ${ }^{16}$ (5) admittance to hospital for amputations because of critical limb ischaemia, prespecified events related to critical limb ischaemia (worsening or increase of ulcers, pain related to peripheral artery disease, necrosis or gangrene, complication caused by amputation of lower limb, infection of skin [ulcer or postamputation wound site], osteomyelitis, procedure related to the ischaemia [eg, revascularisation], wound care [debridement or dressing]), or further diagnostic procedures; and (6) ankle brachial index and toe brachial index.

\section{Safety analysis}

Safety assessments included all adverse and serious adverse events, subjective symptoms, vital signs, resting 12-lead electrocardiographs, ophthalmic examination, and blood tests. The potential safety concerns for growthfactor therapy were occurrence of cancer, cardiovascular ischaemic events, occurrence of active or proliferative retinopathy or neovascularisation in the retina, and renal failure. Occurrence of any of these was noted in the case report form. A history of cancer or an active tumour was an exclusion criterion (panel 1).

An ophthalmic examination (visual acuity, fundoscopy, and slit-lamp microscopy) was done during the 


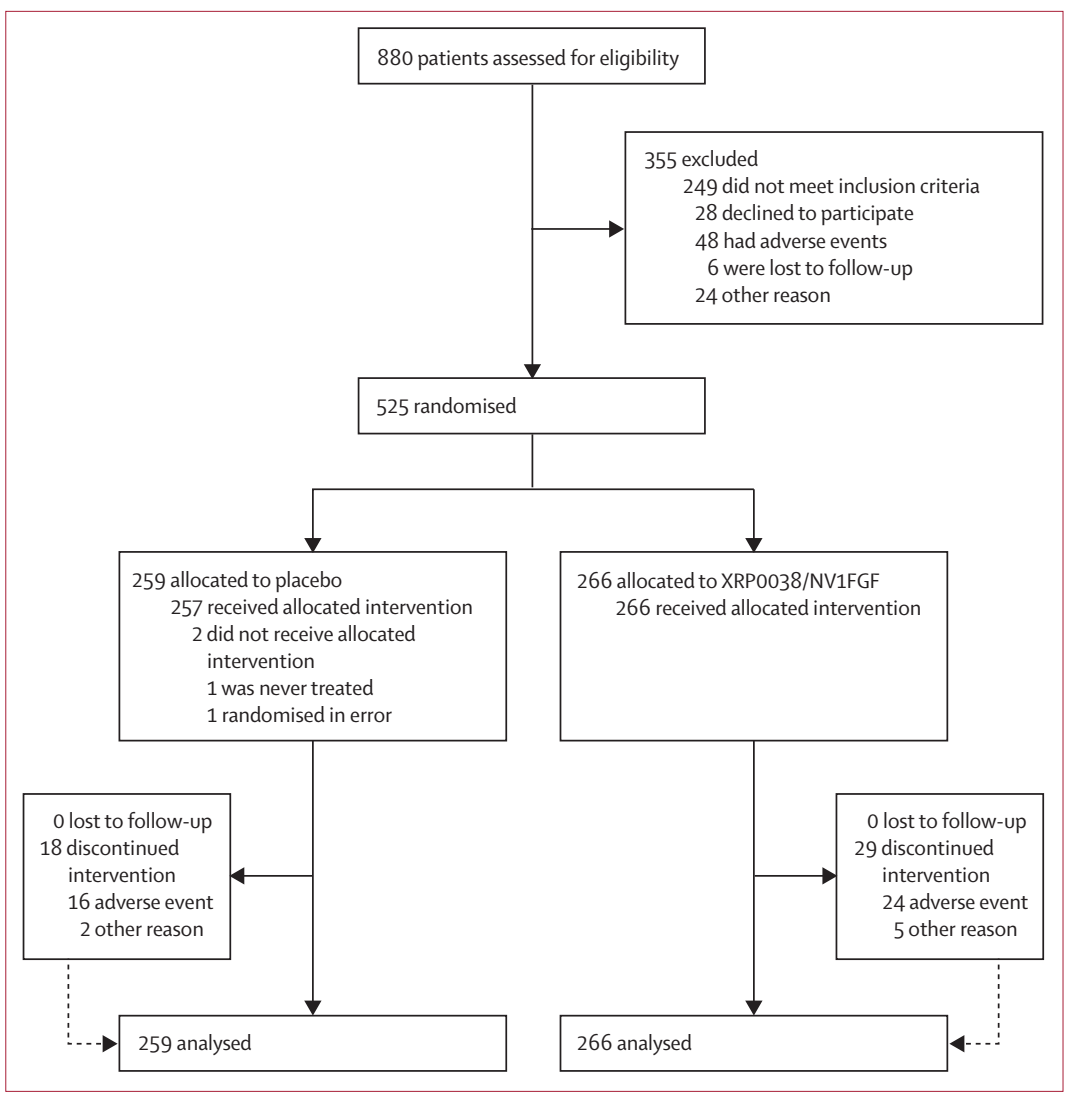

Figure 2: Trial profile

NV1FGF=non-viral 1 fibroblast growth factor.

screening period to exclude active or proliferative retinopathy, or severe macular oedema. The same ophthalmic examinations were repeated at the visits of $1,3,6,9$, and 12 months.

Myocardial infarction, unstable angina, ischaemic stroke, and acute ischaemia of the lower limbs were adjudicated by the events adjudication committee for confirmation of diagnosis. Treatment-emergent adverse events, defined as all adverse and serious adverse events occurring between randomisation and the patient's date of end of study were analysed. All adverse events were coded with the Medical Dictionary for Regulatory Activities.

\section{Statistical analysis}

The calculation of sample size for the combined event rate (amputation and death) was based on the TALISMAN PM201 study ${ }^{16}$ in which a hazard ratio (HR) of 0.558 was recorded. With the assumption of a combined event rate (major amputation or death) at 1 year of at least $35 \%$ in the placebo group, a fixed $\mathrm{HR}$ of $0 \cdot 56$, a fixed patient follow-up of 12 months, and a drop-out rate of 5\%, a sample size of 490 patients ( 245 patients in each treatment group) would give a minimum power of $90 \%$ to show a $44 \%$ reduction in events with NV1FGF compared with placebo, with a twosided log-rank test at a 5\% significance level.
All summaries and statistical analyses were generated with SAS version 8.2 on UNIX environment. All efficacy analyses were done by intention to treat. All safety analyses were based on patients who were treated, according to treatment actually received (treated population). We compared the primary efficacy variable (time to first major amputation of the treated leg above the ankle or to death from any cause) between the two treatment groups using a stratified log-rank test. To examine the relation between treatment effect and subgroups (age, sex, baseline creatinine, gangrene, and ankle brachial index), we analysed the primary efficacy variable using a Cox proportional hazards model in a univariate way with terms for treatment, subgroup, and treatment-by-subgroup interaction. We estimated HRs and 95\% CIs for every subgroup.

We analysed the two secondary efficacy variables (time from randomisation to major amputation or time from randomisation to death) as described above, assessing each of the two parts of the composite primary endpoint separately. We also compared additional secondary efficacy variables (time from randomisation to any amputation, ulcer healing, pain intensity, functionality and health assessment, hospital admission because of critical limb ischaemia, index pressure) between the two groups. The incidence of adverse events was analysed with the Fisher's exact test; relative risks and associated 95\% CIs were estimated.

This trial is registered with ClinicalTrials.gov, number NCT00566657.

\section{Role of the funding source}

A representative of the sponsor was a member of the trial steering committee and was present when decisions were made about trial design. The sponsor was responsible for data monitoring, data collection, and data analysis under the instruction of the trial steering committee. The sponsor of the study had no role in data interpretation or writing of the report. The corresponding author had full access to all the data in the study and had final responsibility for the decision to submit for publication.

\section{Results}

880 patients were screened during a period of 2-8 weeks to check the stability of the skin lesions and to screen for cancer and 525 were randomly assigned (figure 2). Most of these patients were at least 65 years old $(364,69 \%)$, men $(365,70 \%)$, white $(481,92 \%)$, were former or present smokers (321, 61\%), and about 92 (18\%) were obese (defined as a body-mass index $>30 \mathrm{~kg} / \mathrm{m}^{2}$ ). Key baseline patient characteristics were well balanced (table 1). ${ }^{18}$

Other manifestations of atherosclerosis were very frequent, including 234 (45\%) patients with a history of coronary artery disease. Renal impairment, as estimated by a creatinine clearance lower than $50 \mathrm{~mL} / \mathrm{min}$ was present in 152 (29\%), including $44(8 \%)$ patients needing haemodialysis. A history of cancer of more than 5 years before study entry (these patients were eligible) was 
noted in $30(6 \%)$ patients. Of note, those with a history of cancer within the past 5 years were excluded from the study.

258 (49\%) of 525 patients had undergone previous revascularisation procedures of the index leg (either angioplasty or surgery) and 115 (22\%) had a previous minor amputation. 190 (36\%) patients had also undergone a previous revascularisation of the contralateral leg. Additionally, patients who were included had a major impairment in functional status, with only $40 \%$ of patients capable of a daily walking activity $(n=212)$ or unassisted physical performance $(n=220)$.

Except for a higher frequency of previous minor amputations in patients included in North America (21 [31\% of the North American total]) than in the other regions (115 [22\%]), the previous management and the status of the index leg to be treated was similar in all sites across the various regional areas. ${ }^{18}$ Similarly, apart from a higher rate of previous bypass surgery in the non-diabetic population, no major difference in the previous management and status according to the diabetic status was noted. ${ }^{18}$

Unsuitability for revascularisation was retrospectively confirmed for 497 (95\%) patients randomly assigned by the event adjudication committee (webappendix $\mathrm{p} 1$ ) and was related to anatomical criteria (319 patients, 61\%) and an expected low technical success rate of a revascularisation procedure $(487,98 \%)$ of all unsuitable patients. General safety concerns associated with the procedure in patients with a favourable anatomy were present in only eight $(2 \%)$ patients and they did not differ according to geographical area or diabetes status (data not shown).

Almost all patients met the haemodynamic criterion defined at study entry, whereas only a few were enrolled on the basis of a $\mathrm{TcPO}_{2}$ lower than $30 \mathrm{~mm} \mathrm{Hg}$ as a single haemodynamic test to meet the inclusion criterion (table 1).

517 (98\%) patients had infrainguinal arteries affected by stenotic lesions of more than $70 \%$. Thigh arteries were affected in 354 patients (67\%), and arteries below the knee were affected in almost all patients (493, 94\%). Furthermore, multilevel locations of arterial lesions were common, and 495 (94\%) patients had more than one diseased artery. Haemodynamic variables and diseased arterial territories did not differ according to regional area or diabetes status. ${ }^{18}$

Cardiovascular drugs were used in more than $80 \%$ of cases, including $\beta$ blockers, which were given to 242 patients $(46 \%)$ and angiotensin conversion enzyme inhibitors, given to 270 (51\%; webappendix p 2). 341 (65\%) patients took lipid lowering drugs (of whom 328 [62\%] took statins), and 266 (51\%) took antidiabetic drugs. 456 (87\%) took antiplatelet drugs and $225(43 \%)$ anticoagulants. Analgesics were given to $475(90 \%)$ patients, of whom $272(52 \%)$ needed opioids. Non-opioid non-analgesic pain relief drugs (eg, gabapentin, antidepressants) were given

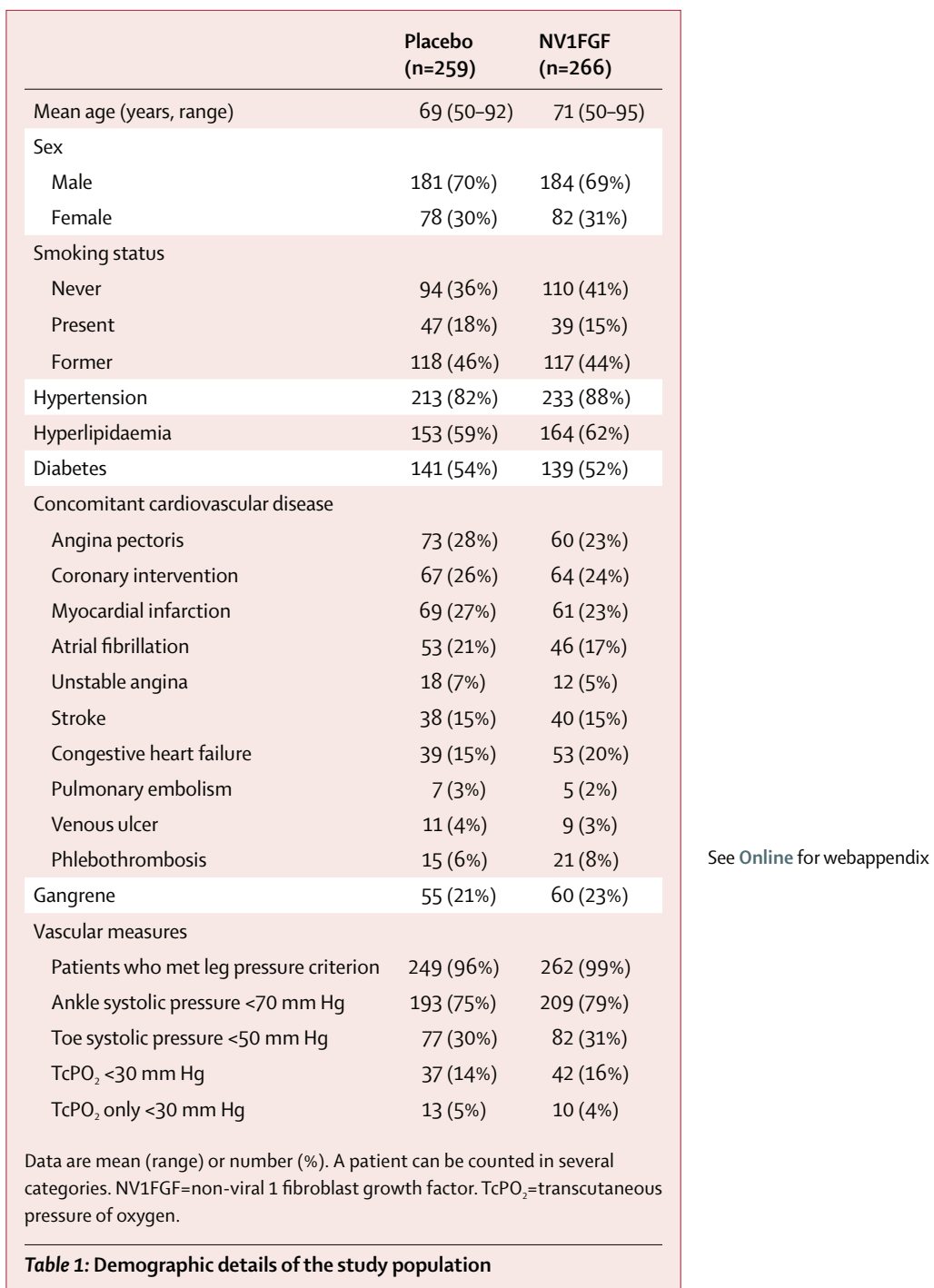

Table 1: Demographic details of the study population

\begin{tabular}{|c|c|c|c|c|c|}
\hline & $\begin{array}{l}\text { Placebo } \\
(n=259)\end{array}$ & $\begin{array}{l}\text { NV1FGF } \\
(n=266)\end{array}$ & $\mathrm{HR}(95 \% \mathrm{Cl})$ & $\operatorname{RR}(95 \% \mathrm{Cl})^{*}$ & $\mathrm{p}$ value \\
\hline \multicolumn{6}{|l|}{ Efficacy } \\
\hline $\begin{array}{l}\text { Major amputation } \\
\text { (above the ankle)/death }\end{array}$ & $86(33 \%)$ & $96(36 \%)$ & $1.11(0.83-1.49)$ &.. & 0.48 \\
\hline Major amputation & $55(21 \%)$ & $67(25 \%)$ & $1 \cdot 20(0.84-1 \cdot 72)$ &.. & 0.31 \\
\hline Death & $39(15 \%)$ & $46(17 \%)$ & $1.15(0.75-1.76)$ &.. & 0.53 \\
\hline \multicolumn{3}{|l|}{ Safety } & \multicolumn{3}{|c|}{.. } \\
\hline Ischaemic heart disease & $26(10 \%)$ & $27(10 \%)$ &.. & $1.00(0.60-1.67)$ & 1.00 \\
\hline Malignant neoplasms & $4(2 \%)$ & $7(3 \%)$ &.. & $1.69(0 \cdot 50-5 \cdot 71)$ & 0.55 \\
\hline Retinal disorders & $15(6 \%)$ & $11(4 \%)$ &.. & $0 \cdot 71(0.33-1.51)$ & 0.42 \\
\hline Renal impairment & $15(6 \%)$ & $20(7 \%)$ & .. & $1.29(0.67-2.46)$ & 0.49 \\
\hline \multicolumn{6}{|c|}{$\begin{array}{l}\text { Efficacy was analysed by intention to treat. Safety was analysed in patients treated ( } 257 \text { patients treated with placebo } \\
\text { and } 266 \text { treated with NV1FGF). NV1FGF=non-viral } 1 \text { fibroblast growth factor. HR=hazard ratio. RR=relative risk. } \\
\text { *Estimated RR. }\end{array}$} \\
\hline
\end{tabular}




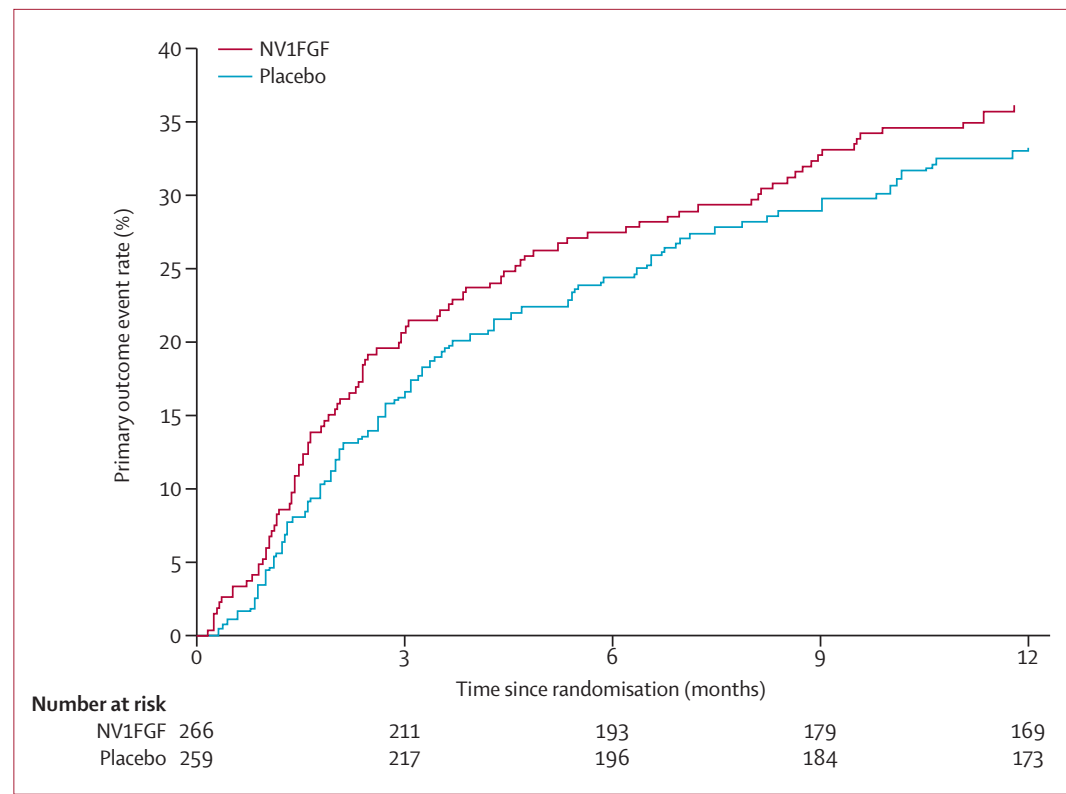

Figure 3: Cumulative incidence curves over time of primary endpoint NV1FGF=non-viral 1 fibroblast growth factor.

\begin{tabular}{|c|c|c|c|c|}
\hline & $\begin{array}{l}\text { Placebo } \\
(n=259)\end{array}$ & $\begin{array}{l}\text { NV1FGF } \\
(n=266)\end{array}$ & $\begin{array}{l}\text { HR } \\
(95 \% \mathrm{Cl})\end{array}$ & $\begin{array}{l}p \text { value for } \\
\text { interaction }\end{array}$ \\
\hline \multicolumn{5}{|c|}{ Age (years) } \\
\hline$<75$ & $52 / 171(30 \%)$ & $57 / 173(32 \%)$ & $1.06(0.73-1.55)$ & 0.883 \\
\hline$\geq 75$ & $34 / 88(39 \%)$ & $39 / 93(42 \%)$ & $1.12(0.70-1 \cdot 79)$ & .. \\
\hline \multicolumn{5}{|l|}{ Sex } \\
\hline Male & $53 / 181(29 \%)$ & $66 / 184(36 \%)$ & $1.36(0.94-1.95)$ & 0.072 \\
\hline Female & $33 / 78(42 \%)$ & $30 / 82(37 \%)$ & $0.78(0.46-1.31)$ &.. \\
\hline \multicolumn{5}{|c|}{ Baseline creatinine clearance $(\mathrm{mL} / \mathrm{min})^{*}$} \\
\hline$<30$ & $14 / 35(40 \%)$ & $20 / 36(56 \%)$ & $1.74(0.84-3.63)$ & 0.970 \\
\hline $30-50$ & $19 / 43(44 \%)$ & $15 / 44(34 \%)$ & $0.79(0.39-1.60)$ & .. \\
\hline $50-80$ & $34 / 85(40 \%)$ & $32 / 90(36 \%)$ & $0.83(0.50-1.37)$ & .. \\
\hline$>80$ & $18 / 85(21 \%)$ & 28/89 (31\%) & $1.51(0.83-2 \cdot 76)$ & .. \\
\hline \multicolumn{5}{|c|}{ Presence of gangrene* } \\
\hline Yes & $30 / 55(55 \%)$ & $33 / 60(55 \%)$ & $1.15(0.69-1.93)$ & 0.9469 \\
\hline No & $56 / 202(28 \%)$ & $63 / 206(31 \%)$ & $1 \cdot 11(0 \cdot 77-1 \cdot 60)$ & .. \\
\hline \multicolumn{5}{|c|}{ Ankle brachial index* } \\
\hline$<0.4$ & $46 / 125(37 \%)$ & $52 / 117(44 \%)$ & $1.23(0.82-1.86)$ & 0.287 \\
\hline $0 \cdot 4-0.79$ & $27 / 80(34 \%)$ & $28 / 103(27 \%)$ & $0.74(0.43-1.26)$ & .. \\
\hline $0.80-0.99$ & $0 / 9(0 \%)$ & $0 / 6(0 \%)$ & .. & .. \\
\hline $1 \cdot 0-1 \cdot 3$ & $2 / 7(29 \%)$ & $1 / 5(20 \%)$ & $0.71(0.04-1.79)$ &.. \\
\hline$>1 \cdot 3$ & $2 / 6(33 \%)$ & $1 / 7(14 \%)$ & .. & .. \\
\hline
\end{tabular}

NV1FGF=non-viral 1 fibroblast growth factor. HR=hazard ratio. *Full set of patients' data not available.

Table 3: Primary endpoint by interaction demographic variables in all randomised patients

to $201(38 \%)$ patients. 14 (5\%) of 259 patients given placebo received intravenous prostaglandin treatment, as did $29(11 \%)$ of 266 patients in the active group (webappendix p 3).

The duration of study treatment was 41 days (range 1-49) days in the placebo group and 40 days (1-52) in the active group. $228(88 \%)$ of 259 patients in the placebo group and $228(86 \%)$ of 266 in the NV1FGF group received four administrations of treatment.

The two groups did not differ significantly in the primary efficacy endpoint of time to major amputation on the treated leg or death, whichever came first (table 2 and figure 3). This endpoint did not vary significantly with the demographic variables (table 3). The time to first major amputation on the treated leg or death was similar between treatment and placebo groups (table 2 and figure 4). Post-hoc primary efficacy analysis on the subgroup of 444 patients (86\%) who received the full course of treatment (four administrations of $4 \mathrm{mg}$ ) shows similar results (data not shown). Table 4 shows causes of death in the two groups.

The main secondary efficacy endpoints (time to first major amputation of the treated leg and time to death) were assessed separately and by the event adjudication committee. Because the primary efficacy endpoint was not significant, no significance could be claimed for the main secondary efficacy endpoints. However, time from randomisation to first major amputation on the treated leg was similar between the NV1FGF group and placebo, and the time from randomisation to any cause of death was similar between the NV1FGF group and placebo (table 2). In view of the large proportion of patients with diabetes, randomisation was stratified by diabetes status. In patients with diabetes, the HR for the primary endpoint was 1.18 (95\% CI $0.79-1.77)$, whereas in patients without diabetes, the HR was $1.01(0.66-1.56$; $\mathrm{p}=0.6796$ for interaction; data not shown).

To inform future trials we continued the analysis for an exploratory additional 6-month review period to see whether any difference in the primary event measures could be detected at this later time, if the primary endpoint was not achieved. This analysis gave no positive result.

Importantly, no patient was lost to follow-up in this trial. The number of patients having treatmentemergent adverse events that occurred after randomisation was similar in both groups: $214(80 \%)$ in the NV1FGF group versus 205 (79\%) in the placebo group. The number of patients with any treatment-emergent adverse event leading to permanent treatment discontinuation was higher in the NV1FGF group (24 of 257 patients who received treatement [9\%]) than in the placebo group (15 of 266 [6\%]). The reasons for discontinuation were infection in seven (44\%) of 16 patients given placebo and 12 (50\%) of 24 active patients who discontinued, and increasing ischaemia (five [33\%] of 15 patients given placebo, five [21\%] of 24 patients given NV1FGF). The safety analysis did not show any significant increase in any treatment-emergent adverse event, particularly for factors previously identified as potential risks associated with gene therapy and angiogenic growth factors, such as proliferative retinopathy or severe macular oedema, acute ischaemic events, and renal impairment (table 2). 


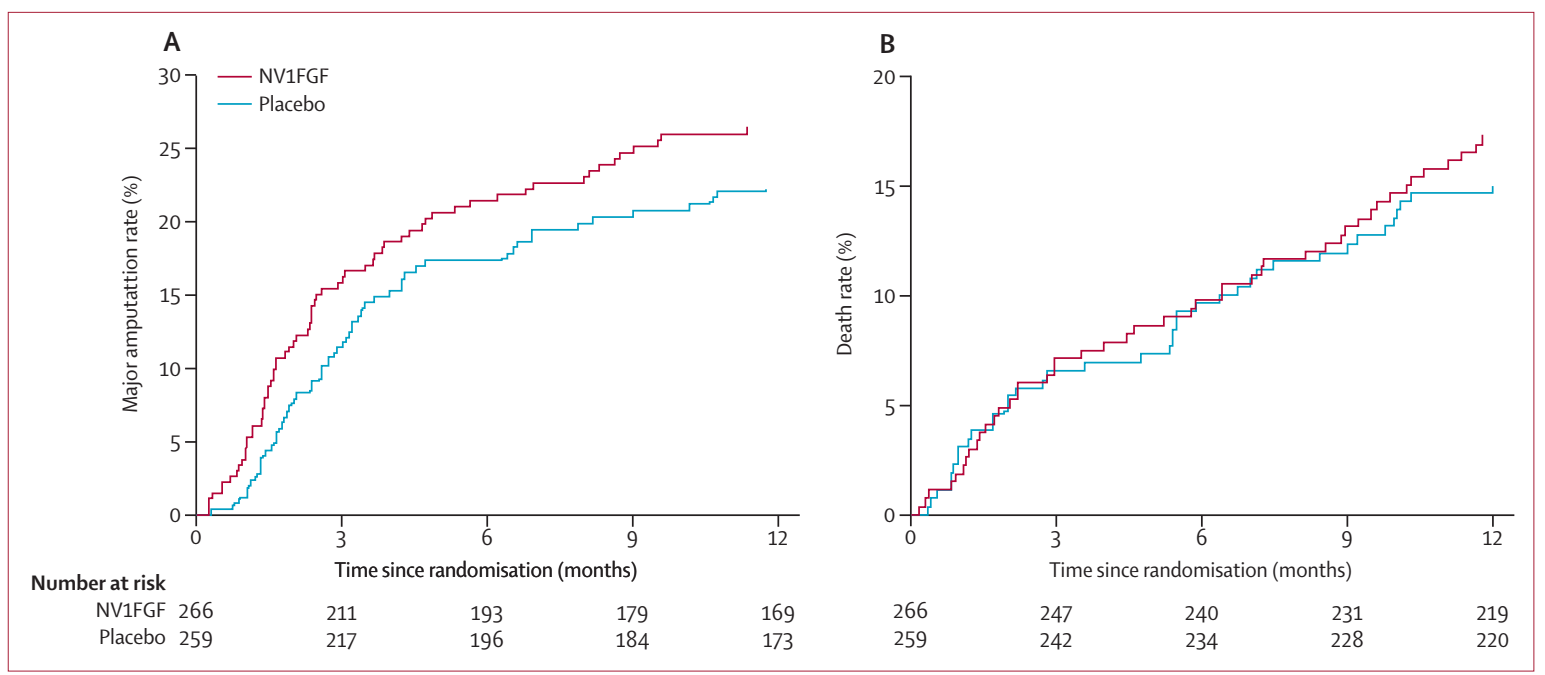

Figure 4: Cumulative incidence curves over time of components of the primary endpoint

(A) First major amputation of the treated leg. (B) Death rate over time. NV1FGF=non-viral 1 fibroblast growth factor.

A higher incidence of treatment-emergent adverse events in the NV1FGF group than in the placebo group was recorded for musculoskeletal and connective tissue disorders system organ class (SOC) (50 [19\%] of 266 vs 25 [10\%] of $257 ; \mathrm{p}=0 \cdot 004]$ mainly represented by pain and discomfort (34 [13\%] of 266 vs 17 [7\%] of 257 ; $=0 \cdot 02$ ) with predominant pain in leg or arm: 26 (10\%) of 266 versus $13(5 \%)$ of $257 \quad(p=0.0057)$. A higher incidence of treatment-related adverse events was also recorded for the metabolism and nutrition disorders SOC (41 [15\%] of 266 versus 19 [7\%] of 257; $\mathrm{p}=0 \cdot 0057)$. This difference was mainly represented by glucose metabolism disorders (including diabetes mellitus): $20(8 \%)$ of 266 versus eight $(3 \%)$ of $257 ; p=0 \cdot 0316$. No proteinuria was reported.

\section{Discussion}

The primary endpoint (fewer deaths or first major amputation of the treated leg whichever came first) was not achieved, nor were the secondary endpoints. This result contrasts with the phase 2 TALISMAN study in which significant benefit was recorded in the secondary endpoint (major amputation) and in the combined endpoint (major amputation or death, whichever came first).

Examination of the populations studied in the two trials showed no clear evidence of any baseline differences that could have caused the discrepancy in the results. Similar inclusion and exclusion criteria were required for both studies (in addition to the similar design, treatment dosing, and regimen used in TAMARIS to replicate phase 2 results). The phase 3 trial had about $10 \%$ more patients with diabetes than did the phase 2 trial, but a subgroup analysis of non-diabic patients alone did not change the results. Furthermore, TAMARIS included patients with end-stage renal disease (by contrast with

\begin{tabular}{|lcc|}
\hline & Placebo $(\mathbf{n = 2 5 9 )}$ & NV1FGF $(\mathbf{n = 2 6 6})$ \\
\hline All-cause mortality & $39(15 \%)$ & $46(17 \%)$ \\
Cardiovascular death & $18(7 \%)$ & $20(8 \%)$ \\
Sudden death & $1(<1 \%)$ & $5(2 \%)$ \\
Unknown cause & $4(2 \%)$ & $9(3 \%)$ \\
Non-cardiovascular death & $16(6 \%)$ & $12(4 \%)$ \\
Data analysed by intention to treat. NV1FGF=non-viral 1 fibroblast \\
growth factor.
\end{tabular}

TALISMAN). However, assessment of data with removal of these subgroups did not change the results. Nor did geographical distribution of study sites contribute to the differences recorded. When the analysis was restricted to the TALISMAN sites only, the results were still negative.

A key observation, however, was that the major amputation or death rate in the placebo group at 360 days in TALISMAN (more than 50\%) differed from that in the placebo group in TAMARIS (33\%). By contrast, the treated-group endpoint rates were similar in both studies, suggesting that the significance achieved in TALISMAN depended on a high placebo endpoint rate. It is unlikely that medical or background treatment improved in the 4 years between studies, since assessment of the baseline characteristics suggests similar use of statins and other vascular risk modifiers in the two studies. Geographical variation in event rates between studies could exist, although the low number of patients included in some regions might not support the conclusion that the observed placebo rates indicate the disease management in these countries. In the TAMARIS placebo group, the primary endpoint ranged from $20 \%$ in Latin America to $48 \%$ in North America. The TALISMAN study was done in Europe and had only 56 patients randomly assigned to 
Panel 2: Research in context

\section{Systematic review}

We searched PubMed and Cochrane Library databases up to

March 9, 2011, without language restrictions for articles reporting randomised trials, systematic reviews, and meta-analyses with the search terms "peripheral vascular disease", "peripheral artery disease", "gene therapy", and "angiogenesis". We excluded articles that included non-atherosclerotic symptoms of peripheral artery disease, such as thromboangiitis obliterans, and excluded trials of cell-based therapies. Our search identified two meta-analyses and three systematic reviews. ${ }^{11,16,19-22}$ Additionally, we identified one report in press about the use of gene therapy in claudication.

\section{Interpretation}

The meta-analyses, reviews, and the report currently in press assessed several angiogenic methods, including proteins and genes delivered with a viral vector or as a plasmid. The routes of administration were intra-arterial and intramuscular. In the treatment of claudication, in which the primary endpoint was improvement in peak exercise performance, gene therapy had no benefit. In critical limb ischaemia, benefit was noted in some studies for several surrogate endpoints such as improved limb vascularity, transcutaneous oxygen content, ulcer healing, or relief of pain. These results were not consistent between studies. In one trial, ${ }^{16}$ amputation-free survival was improved with NV1FGF. ${ }^{16}$ However, overall, results from the meta-analyses and systematic reviews showed no benefit for gene therapy on risk of amputation or death.

NV1FGF=non-viral 1 fibroblast growth factor

placebo. Moreover, infection-related amputations were more common in the placebo group of TALISMAN ${ }^{16}$ than in the placebo group of TAMARIS. Thus, the ability to estimate a true untreated event rate in this population is very challenging.

Analyses of the data did not show any selection bias within the two groups since they were well matched for baseline characteristics. Because the event rate (major amputation or death) was lower than expected in the placebo group, it could be argued that the wrong patient population was selected, and that more severe disease should have been studied. However, no trend to efficacy was detected in the most ischaemic patients (table 3 shows results for presence of gangrene).

No clear adverse safety signals were noted with NV1FGF. Safety analyses showed no difference in cardiovascular events or death between active and placebo groups. The previously identified potential risks associated with gene therapy and angiogenic growth factors include proliferative retinopathy, malignancy, macular oedema, acute ischemic events, and renal impairment. Thus, a long-term safety follow-up of 36 months has been implemented and is still in progress.
This follow-up is in line with the European Medicinal Agency (EMEA) guidance for long-term safety assessment of patients treated with gene therapy products.

In conclusion, results from this large gene-therapy study in critical limb ischaemia to be completed to date (panel 2), portray the challenges faced by the development programmes of single genes, such as extrapolation from conclusive animal studies to define the optimum dose, vector, route, and duration of administration, as well as whether the administration of any one gene could result in therapeutic angiogenesis leading to the prevention of limb amputations.

\section{Contributors}

JB and WRH formed the core writing team but all the authors contributed equally to study design, study management, data interpretation, data collection, data analysis, and writing of the report.

\section{TAMARIS steering committee}

Jill Belch, William R Hiatt, Iris Baumgartner, I Vickie Driver, Sigrid Nikol, Lars Norgren, and Eric Van Belle.

\section{Conflicts of interest}

All authors are members of the steering committee of the TAMARIS trial and receive an honorarium from Sanofi-Aventis, Paris, France.

\section{Acknowledgments}

This study was funded by Sanofi-Aventis, Paris, France. We thank Soraya Bekkali from Sanofi-Aventis, Paris, France, for the coordinating and oversight activities of her and her team.

\section{References}

1 Baumgartner I, Schainfeld R, Graziani L. Management of peripheral vascular disease. Annu Rev Med 2005; 56: 249-72.

2 Patterson D, Belch JJF. Pathophysiology of critical limb ischemia. In: Creager MA, Dzau VJ, Loscalzo J, eds. Vascular medicine: a companion to Braunwald's heart disease. Oxford: Saunders Elsevier 2006: 248-53.

3 Dormandy J, Heeck L, Vig S. The fate of patients with critical leg ischemia. Semin Vasc Surg 1999; 12: 142-47.

4 Dormandy JA, Rutherford RB. Management of peripheral arterial disease (PAD). TASC Working Group. TransAtlantic Inter-Society Consensus (TASC). J Vasc Surg 2000; 31 (1 Pt 2): S1-S296.

5 Treat-Jacobson D, Halverson SL, Ratchford A, Regensteiner JG, Lindquist R, Hirsch AT. A patient-derived perspective of health related quality of life with peripheral arterial disease. J Nurs Scholarship 2002; 34: 55-60.

6 Albers M, Fratezi AC, DeLuccia N. Assessment of quality of life of patients with severe ischemia as a result of infrainguinal arterial occlusive disease. J Vasc Surg 1992; 16: 54-59.

7 Ischemia EWGoCL. Second European consensus document on chronic critical leg ischemia. Circulation 1991; 84 (4 suppl): IV1-26.

8 Halperin JL. Evaluation of patients with peripheral vascular disease. Thromb Res 2002; 106: 303-11.

9 Ruffolo AJ, Romano M, Ciapponi A. Prostanoids for critical limb ischaemia. Cochrane Database Syst Rev 2010; 1: CD006544.

10 Norgren L, Hiatt WR, Dormandy JA, Nehler MR, Harris KA, Fowkes FG. Inter-Society Consensus for the Management of Peripheral Arterial Disease (TASC II). J Vasc Surg 2007; 45 (Suppl): S5-67.

11 De Haro J, Lopez-Quintana FAA, Martinez-Aguilar AFE, Varela C. Meta-analysis of randomized, controlled clinical trials in angiogenesis: gene and cell therapy in peripheral arterial disease. Heart Vessels 2009; 24: 321-28.

12 Caron A, Michelet S, Caron A, et al. Human FGF-1 gene transfer promotes the formation of collateral vessels and arterioles in ischemic muscles of hypercholesterolemic hamsters. J Gene Med 2004; 6: 1033-45.

13 Hershey JC, Corcoran HA, Baskin EP, et al. Enhanced hindlimb collateralization induced by acidic fibroblast growth factor is dependent upon femoral artery extraction. Cardiovasc Res 2003; 59: 997-1005. 
14 Baumgartner I, Chronos N, Comerota A, et al. Local gene transfer and expression following intramuscular administration of FGF-1 plasmid DNA in patients with critical limb ischemia. Mol Ther 2009; 17: 914-21.

15 Comerota AJ, Throm RC, Miller KA, et al. Naked plasmid DNA encoding fibroblast growth factor type 1 for the treatment of end-stage unreconstructible lower extremity ischemia: preliminary results of a phase I trial. J Vasc Surg 2002; 35: 930-36.

16 Nikol S, Baumgartner I, Van Belle E, et al. Therapeutic angiogenesis with intramuscular NV1FGF improves

amputation-free survival in patients with critical limb ischemia. Mol Ther 2008; 16: 972-78.

17 Katz J, Melzack R. Measurement of pain. Surg Clin North Am 1999; 79: 231-52.

18 Van Belle E, Nikol S, Norgren L, et al, TAMARIS steering committee. A randomized, double-blind, placebo-controlled gene therapy study using NV1FGF for prevention of amputation and death in critical limb ischemia (TAMARIS). Rationale, design and baseline patient characteristics. Eur J Endovasc Vasc Surg (in press).
19 Ghosh R, Walsh SR, Tang TY, Noorani A, Hayes PD. Gene therapy as a novel therapeutic option in the treatment of peripheral vascular disease: systematic review and meta-analysis. Int J Clin Pract 2008; 62: $1383-90$

20 Gabhann FM, Annex BH, Popel AS. Gene therapy from the perspective of systems biology. Curr Opin Mol Ther 2010; 12: 570-77.

21 Gupta R, Tongers J, Losordo DW. Human studies of angiogenic gene therapy. Circ Res 2009; 105: 724-36.

22 Germani A, Di Campli C, Pompilio G, Biglioli P, Capogrossi MC. Regenerative therapy in peripheral artery disease. Cardiovasc Ther 2009; 27: 289-304. 